\title{
Associação entre número de consultas pré-natal e as características maternas e neonatais
}

\author{
Association between number of prenatal consultations and maternal and neonatal characteristics \\ Asociación entre número de consultas prenatales y características maternas y neonatales
}

Iara do Nascimento Pantoja ${ }^{1}$, Cleise Ellen Ferreira Pantoja ${ }^{1 *}$, Glenda Roberta Oliveira Naiff Ferreira ${ }^{1}$, Rubenilson Caldas Valois ${ }^{2}$, Hallessa de Fátima da Silva Pimentel ${ }^{3}$, Gilvana de Carvalho Moraes $^{3}$, Nazaré do Socorro Oliveira Afonso', Gabriela Faias de Lima1', Ana Paula Loureiro de Brito $^{1}$, Gyselle Morais da Silva ${ }^{3}$.

\section{RESUMO}

Objetivo: Analisar a influência das características sociodemográficas no número de consultas realizadas no pré-natal e como essas influenciam nos desfechos maternos e neonatais. Métodos: Trata-se de um estudo transversal e analítico sobre os fatores que influenciam e são influenciados pelo número de consultas prénatal ocorridas no ano de 2017 nos municípios de Bragança, Capanema e Viseu. Os dados foram extraídos da base do Sistema de Informação de Nascidos Vivos (SINASC), no qual foram realizadas estatísticas descritivas e analíticas. Resultados: Foi registrado um total de 4.279 nascidos vivos nos municípios do estudo, sendo 49\% em Bragança, 26,7\% em Viseu e 24,3\% em Capanema. Apresentaram associação com o número de consultas estatisticamente significativa: o estado civil em Viseu e Capanema; idade de 10 a 17 anos em Capanema; a escolaridade em todos os municípios. Demonstraram associação $(p<0,05)$ do tipo de parto em Braqança e Capanema; o tempo de gestação em todos os municípios; o peso em Bragança e Viseu; e o apgar em Capanema. Conclusão: Um acompanhamento pré-natal de qualidade pode ajudar na identificação precoce de intercorrências gestacionais contribuindo na redução de riscos, tanto para a gestante quanto para o concepto, tornando-se imprescindível para a promoção da saúde materna e neonatal.

Palavras-chave: Pré-natal, Consulta, Recém-nascido.

\begin{abstract}
Objective: To analyze the influence of sociodemographic characteristics on the number of prenatal consultations and how they influence maternal and neonatal outcomes. Methods: This is a cross-sectional and analytical study on the factors that influence and are influenced by the number of prenatal consultations that took place in 2017 in the municipalities of Bragança, Capanema and Viseu. The data were extracted from the basis of the Live Birth Information System (SINASC), in which descriptive and analytical statistics were performed. Results: A total of 4,279 live births were recorded in the municipalities of the study, $49 \%$ in Bragança, $26.7 \%$ in Viseu and $24.3 \%$ in Capanema. They were associated with a statistically significant number of consultations: marital status in Viseu and Capanema; age 10 to 17 years in Capanema; schooling in all municipalities. They demonstrated an association $(p<0.05)$ of the type of delivery in Bragança and Capanema; the length of pregnancy in all municipalities; the weight in Bragança and Viseu; and apgar in Capanema. Conclusion: A quality prenatal care can help in the early identification of pregnancy complications, contributing to the reduction of risks, both for the pregnant woman and for the fetus, making it essential for the promotion of maternal and neonatal health.
\end{abstract}

Keywords: Prenatal, Consultation, Newborn.

RESUMEN

Objetivo: Analizar la influencia de las características sociodemográficas en el número de consultas prenatales y cómo influyen en los resultados maternos y neonatales. Métodos: Se trata de un estudio transversal y analítico sobre los factores que influyen y son influenciados por el número de consultas

\footnotetext{
1 Universidade Federal do Pará (UFPA), Belém - PA. *E-mail: ecleise@yahoo.com.br

2 Universidade Estadual do Pará (UEPA), Belém - PA.

3 Universidade da Amazônia (UNAMA), Belém - PA.
} 
prenatales que tuvieron lugar en 2017 en los municipios de Bragança, Capanema y Viseu. Los datos se extrajeron de la base del Sistema de Información de Nacimientos Vivos (SINASC), en el cual se realizaron estadísticas descriptivas y analíticas. Resultados: Se registraron un total de 4.279 nacidos vivos en los municipios del estudio, 49\% en Bragança, 26,7\% en Viseu y 24,3\% en Capanema. Se asociaron a un número de consultas estadísticamente significativo: estado civil en Viseu y Capanema; de 10 a 17 años en Capanema; escolaridad en todos los municipios. Demostraron una asociación $(p<0.05)$ del tipo de parto en Bragança y Capanema; la duración del embarazo en todos los municipios; el peso en Bragança y Viseu; y apgar en Capanema. Conclusión: Una atención prenatal de calidad puede ayudar en la identificación temprana de las complicaciones del embarazo, contribuyendo a la reducción de riesgos, tanto para la gestante como para el feto, por lo que es fundamental para la promoción de la salud materna y neonatal.

Palabras clave: Prenatal, Consulta, Recién nacido.

\section{INTRODUÇÃO}

A saúde materna tem impacto na saúde pública, por ainda ser uma preocupação global, no qual a saúde e bem-estar devem ser prioritários desde o momento do início da gestação, interferindo na qualidade do pré-natal, pré-parto, parto e pós-parto e na vida do Recém-Nascido (SILVA A, et al., 2021). Apesar da progrssiva implementação das boas práticas que abrangem a saúde da mulher, mortes e doenças correlacionadas à gravidez ainda estão extremamente eminentes (MINISTÉRIO DA SAÚDE, 2015).

$\mathrm{Na}$ literatura cientifica, ainda observamos dados quantitativos acentuados de mortalidade materna relacionadas a diversos fatores enfatizando as urgencias obstétricas. A mortalidade materna é um indicador de qualidade dos serviços de saúde oferecidos a gestante, principalmente no sistema público, no qual encontramos diversas mulheres em situações com baixo teor aquisitivo, que interfere no período gestacional. Além disso, as desigualdades geográficas também contribuem para desfechos negativos da gravidez, relacionando-se a dificuldade de acesso e muitas vezes á determinadas regiões que apresentam precariedade nas práticas de pré-parto, parto e pós-parto. (MARTINS A e SILVA L, 2018).

A assistência pré natal deve acolher e assegurar o desenvolvimento em todas as fases da gestação, as transformações físicas e emocionais, sem impacto para saúde materna e neonatal, desta forma é necessário que seja garantida que a gestante tenha acesso ao número de consultas preconizado, iguais ou superior a 6 consultas (MINISTÉRIO DA SAÚDE, 2016). O pré natal é uma ferramenta que busca sistematizar o cuidado á gestante, garantindo assistencia materno infantil, no qual a equipe multiprofissional irá garantir a mulher o direito de emponderamento sobre o seu próprio corpo e cuidado ao seu recémnascido, além disso, também vai buscar prevenir situações de alto risco, no intuito de reduzir a morbimortalidade materno-infantil (FESCINAR R, et al, 2011).

Além disso, é importante frisarmos que as mudanças fisiológicas que a mulher passa, percebemos uma mudança de cenário, no qual se faz necessário a incorporação de diversas condutas que envolvam a humanização; o desenvolvimento de ações educativas e preventivas, da detecção precoce de patologias e identificação de risco gestacional (OLIVEIRA E, et al., 2016). A ampliação da assistencia pré-natal e atenção aos cuidados no purpério e com o Recém-Nascido nos paises em desenvolvimento vem ajudando a diminuir as taxas de mortalidade materno-infantil nos últimos anos, visto que nos leva a uma reflexão sobre o conhecimento cientifico e tecnológico já existente e a partir desse principio, se torna possivel identificar fatores de risco relacionados com a mortalidade materno-infantil (BORGES M, et al., 2019)

Pesquisas mostram que para entendermos o contexto destas mulheres, deve-se haver dados nacionais coerentes que possam mostrar a atual cobertura do pré-natal para compreensão da avaliação prestada. Os indicadores são instrumentos que devem refletir as falhas que precisam ser mudadas e os avanços na assistência, como uma das formas de melhorar o caminho na qualidade desse pré-natal (VIELLAS E, et al., 2014).

De acordo com o programa da rede cegonha, é relevante ampliar-se o atendimento e trazer melhorias as mulheres como um novo modelo de atenção a saúde da mulher, uma vez que a rede busca organizar o fluxo do atendimento á essa mulher, respeitando e garantindo seus direitos, como a garantia de acesso ao Sistema Único de Saúde, para acompanhamento pré-natal (SOUZA K, et al., 2021). 
Existem diversos fatores que influenciam a não realização do pré-natal, principalmente os fatores socioeconômicos e geográficos, o que limita o serviço assistencial. No entanto, existe um fator que é importante enfatizar que é quanto à rede de apoio em que a gestante está inserida, pois a importância do apoio familiar influencia no desejo de participar das consultas do pré-natal (TITALEY R, et al., 2016).

Certamente, diante da literatura científica, observamos que um bom pré-natal influencia em uma gravidez desejada e com bons desfechos para a mulher e o recém-nascido. Porém, observamos também que o vínculo com os serviços de saúde também é peça fundamental, para que haja uma maior adesão das mulheres aos serviços prestados, pois no cenário atual, o SUS disponibiliza testes sorológicos, além de outros exames adicionais, cobertura para as gestantes de alto risco, entre outros serviços (DARMONT R, et al., 2015).

A baixa cobertura de atenção primária, como tendo reflexos no pré-natal, a gestante que não faz a consulta completa tem piores condições socioeconômicas, e pode ter intercorrência durante 0 parto e as maternidades de alto risco nem sempre estão próximas ao local de moradia, que pode demorar o trabalho de parto e ter como consequência desfechos negativos para a mãe e o concepto (FERRAZ $L$ e BORDIGNON M, 2015)

A Atenção Básica é a porta de entrada para a gestante, onde se pode prevenir complicações através de uma assistência de pré-natal adequada, garantindo recursos necessários para um percurso de período gestacional saudável, e a partir do momento que essa mulher cria um vinculo com este ambiente, a atenção primária está garantindo, através da rede cegonha, uma atenção humanizada, garantindo e respeitando os direitos da mulher, além de apresentar a participação no processo de captação das gestantes desde o primeiro trimestre (ARAÚJO C, et al., 2017)

No entanto, ainda existem locais com baixa cobertura de 7 consultas, como em áreas do estado do Pará. Entre 2008 a 2017 foram registrados 92.729 nascidos vivos, na região de Rios Caetés. A taxa de cobertura pré-natal de acordo com o número de consultas demonstra que $13,2 \%$ (12.284) não realizaram nenhuma consulta; $14,4 \%$ (13.307) realizaram entre 1 a 3 consultas; 45, 2\% (41.893) realizaram de 4 a 6 consultas e 26,8\% (24.872) com 7 ou mais consultas (MINISTÉRIO DA SAÚDE, 2016).

As principais complicações do parto, estão muitas vezes entrelaçadas a falta de qualidade do pré-natal, no qual tem papel importante na prevenção e/ou detecção precoce de doenças que podem ser evitadas. De acordo com o Ministério da Saúde, um pré-natal de qualidade deve ser iniciado até o quarto mês de gestação, ter no mínimo seis consultas de acompanhamento multiprofissional, além da realização de testes de sorologias e laboratoriais. Além disso, destaca-se que o programa Rede cegonha, lançado em 2011 traz como principais objetivos assegurar uma rede de cuidados de atenção humanizada, que inclui o pré natal e parto, para que se tenha desfechos positivos em saúde, tanto para a mulher, quanto para o RN (NASCIMENTO I, et al., 2017; MENDES R, et al., 2019).

Os indicadores que mostram as caracteristicas maternas e neonatais, conseguem demonstrar o contexto em que essas mulheres estão inseridas, o acesso aos serviços de saúde e as possiveis falhas nas organizações de saúde. São capazes de mostrar também as limitações quanto a ampliação e a cobertura da clientela (MENDES R, et al., 2019).

Diante desta problemática, este estudo teve como objetivo analisar a influência das características sociodemográficas no número de consultas realizadas no pré-natal e como essas influenciam nos desfechos maternos e neonatais, como forma de trazer estudos recentes que leva a reflexão de possiveis melhorias para este público.

\section{MÉTODOS}

Trata-se de um estudo transversal e analítico sobre os fatores que influenciam e são influenciados pelo número de consultas pré-natal ocorridas no ano de 2017, em residentes dos municípios de Bragança, Capanema e Viseu, localizados na Região de Saúde dos Caetés, componentes da macrorregião II, estado 
do Pará. Os dados foram coletados entre em julho de 2019, sendo extraídos da base do Sistema de Informação de Nascidos Vivos (SINASC), no sítio eletrônico do Departamento de Informática do Sistema Único de Saúde SUS (Datasus), com utilização do programa Tabwin 4.1.5 para extração do banco de dados na íntegra.

Foram selecionados apenas os dados de nascidos vivos de residentes dos municípios da Região de Caetés, sendo selecionados os municípios que tiveram acima de 1.000 nascimento no ano de 2017 . Após a extração, foram selecionadas as variáveis de interesse, e em seguida foram decodificadas e armazenadas em planilha do programa Microsoft Excel.

A variável número de consultas foi agrupada em dois grupos: de 0 a 3 consultas (evento) e 4 a 7 consultas (padrão). As variáveis sociodemográficas foram: estado civil, casado/união estável (padrão/referência) e solteiro/separado/viúvo (evento); idade, 10 a 17 anos (evento), 18 a 29 anos (evento) e 30 e mais (padrão/referência); escolaridade: 1 a 7 anos de estudo (evento) e 8 a 12 anos e mais (padrão/referência). As variáveis dos desfechos maternos foram: tipo de parto, cesáreo (evento) e vaginal (padrão); tempo de gestação, prematuridade/pré-termo de 22 a 36 semanas (evento) e a termo de 37 a 41 semanas (padrão). Os desfechos neonatais foram: peso ao nascer, baixo peso, quando inferior a 2500 gramas (evento) e normal quando peso igual ou superior a 2500 gramas (padrão); apgar no $1^{\circ}$ minuto, dificuldade (evento) e normal (padrão).

Para testar a primeira hipótese, a influência das características sociodemográficas maternas em relação ao número de consultas, as variáveis sociodemográficas maternas (escolaridade, idade da mãe e estado civil) foram consideradas variáveis independentes e o número de consultas como dependentes. Para testar a segunda hipótese, se o número de consultas tem influência nos desfechos maternos e neonatais, a variável consulta foi considerada como independente e as variáveis desfechos maternos (tipo de parto e tempo de gestação) e neonatais (peso e apgar) foram consideradas como dependentes.

Foram realizadas estatísticas descritivas média, desvio padrão (DP), e intervalo de confiança (C) e analíticas. Para testar as hipóteses foi realizado o teste do qui-quadrado para verificar a existência de associação entre as variáveis dependente e independentes, por meio do programa Bioestat (versão 5.3). As variáveis dependentes que apresentaram $p<0,05$ foram codificadas ( 0 para padrão e 1 para o evento) para realização da regressão logística binária no programa Minitab $18 \circledast$. Foram considerados os valores de Odds ratio, coeficiente e valor de $p<0,05$.

Os pesquisadores não tiveram contato com as pessoas que internaram, uma vez que foram utilizadas fontes de dados secundários de domínio público, desta forma sendo dispensado de apreciação por Comitê de Ética em Pesquisa, conforme recomenda o parágrafo único do artigo 1ํ da Resolução № 510, de 07 de abril de 2016, do Conselho Nacional de Saúde (CNS). Os pesquisadores respeitaram todas as diretrizes éticas de pesquisa com seres humanos, conforme recomenda a Resolução do CNS, $n^{\circ} 466$, de 12 de dezembro de 2012.

\section{RESULTADOS}

\section{Associação entre características sociodemográficas maternas e o número de consultas pré-natal}

Para analisar se as características sociodemográficas maternas influenciam no número de consultas prénatal, inicialmente, foi realizado o teste do qui-quadrado e todas as variáveis que apresentam associação estatística, com $p<0,05$, foram então submetidas a regressão logística binária.

Foi registrado um total de 4.279 nascidos vivos nos municípios do estudo, sendo 2.099 (49\%) em Bragança, 1.140 (26,7\%) em Viseu e 1.040 (24,3\%) em Capanema. Entre as características sociodemográficas maternas, a média de idade foi de 23,98 anos (DP $\pm 6,2$; IC 95\%: 23,7 - 24,1). Observouse que o estado civil de $2.075(48,75 \%)$ era de solteiras, $1.702(39,99 \%)$ de união estável, $474(11,14 \%)$ casados, $03(0,07 \%)$ separadas, $02(0,05 \%)$ viúvas e 23 não possuíam informação. Considerando a escolaridade, 249 (6\%) tinham entre 1 a 3 anos de estudo; 1.311 (31,5\%) de 4 a 7 anos; 2.314 (55,5\%) de 8 a 11 anos; 294 (7\%) de 12 a mais anos de estudo e 99 não possuíam informação. 
Na Tabela 1 estão descritos os resultados do teste do qui-quadrado para cada município estudado. Apresentaram associação estatisticamente significativa: o estado civil em Viseu e Capanema; idade de 10 a 17 anos em Capanema; a escolaridade em todos os municípios.

Tabela 1 - Associação entre características sociodemográficas maternas e número de consultas pré-natal nos municípios de Bragança, Capanema e Viseu. 2017.

\begin{tabular}{|c|c|c|c|c|c|c|c|c|c|c|c|c|}
\hline \multirow{3}{*}{$\begin{array}{l}\text { Características } \\
\text { sociodemográficas } \\
\text { maternas }\end{array}$} & \multicolumn{12}{|c|}{ Número de consultas por município } \\
\hline & \multicolumn{4}{|c|}{ Bragança (2099) } & \multicolumn{4}{|c|}{ Viseu (1140) } & \multicolumn{4}{|c|}{ Capanema (1040) } \\
\hline & $\begin{array}{l}0 \text { a } 3 \\
744\end{array}$ & $\begin{array}{l}4 \text { a } 7 \\
1355\end{array}$ & $x^{2}$ & $p$ & $\begin{array}{l}0 \text { a } 3 \\
293\end{array}$ & $\begin{array}{l}4 \text { a } 7 \\
847\end{array}$ & $x^{2}$ & $P$ & $\begin{array}{l}0 \text { a } 3 \\
400\end{array}$ & $\begin{array}{r}4 \text { a } 7 \\
640\end{array}$ & $x^{2}$ & $p$ \\
\hline \multicolumn{13}{|l|}{ Estado civil } \\
\hline Casado/ União Estável & 323 & 610 & & & 152 & 156 & & & 135 & 400 & & \\
\hline $\begin{array}{l}\text { Solteiro/ Separado/ } \\
\text { Viúvo }\end{array}$ & 420 & 737 & 0.566 & 0.452 & 141 & 280 & 16.2 & $<0.0001$ & 263 & 239 & 79.6 & $<0.0001$ \\
\hline $\mathrm{SI}^{*}$ & 1 & 8 & & & & & & & & & & \\
\hline \multicolumn{13}{|l|}{ Idade } \\
\hline 10 a 17 anos & 103 & 210 & 0.189 & 0.664 & 62 & 144 & 0.89 & 0.34 & 58 & 62 & 8.6 & 0.003 \\
\hline 18 a 19 anos & 489 & 859 & 0.29 & 0.59 & 185 & 567 & 0.01 & 0.92 & 267 & 417 & 3.6 & 0.056 \\
\hline$\geq 30$ anos & 152 & 286 & & & 46 & 136 & & & 75 & 161 & & \\
\hline \multicolumn{13}{|l|}{ Escolaridade } \\
\hline 1 a 7 anos & 273 & 440 & & & 156 & 386 & & & 143 & 162 & & \\
\hline$\geq 8$ anos & 458 & 906 & 4.352 & 0.037 & 115 & 445 & 9.6 & 0.001 & 224 & 460 & 17.4 & $<0.0001$ \\
\hline $\mathrm{SI}^{*}$ & 13 & 9 & & & 22 & 16 & & & 33 & 18 & & \\
\hline
\end{tabular}

Fonte: Pantoja IN, et al., 2021.

Na regressão logística os valores OR abaixo de 1 foram associados a ter realizado de 4 a 7 consultas, enquanto os valores de OR acima de 1 foram associados de 0 a 3 consultas pré-natal. Os resultados demonstraram que mães com estado civil solteira/separada/viúva procedentes de Viseu têm três vezes mais chances de realizaram um menor número de consultas, enquanto em Capanema essas chances diminuem para cerca de duas vezes. As mães com idade entre 10 a 17 anos procedentes de Capanema tem cerca de duas vezes mais chances de realizarem de 0 a 3 consultas. Já a escolaridade de 1 a 7 anos é um fator que influencia ao menor número de consultas pré-natal (Tabela 2).

Tabela 2 - Regressão logística binária entre características sociodemográficas maternas e número de consultas pré-ntal nos municípios de Bragança, Capanema e Viseu. 2017.

\begin{tabular}{|c|c|c|c|c|c|c|c|c|c|}
\hline \multirow{2}{*}{ Variáveis } & \multicolumn{3}{|c|}{ Bragança } & \multicolumn{3}{|c|}{ Viseu } & \multicolumn{3}{|c|}{ Capanema } \\
\hline & OR $^{\star}$ & IC 95\%** & $P$ & OR & IC 95\% & $P$ & OR & IC 95\% & $p$ \\
\hline \multicolumn{10}{|l|}{ Estado Civil } \\
\hline $\begin{array}{l}\text { Solteiro/ } \\
\text { separado/ viúvo }\end{array}$ & - & - & - & 3,26 & $2,51-4,24$ & 0 & 1,84 & $1,41-2,41$ & 0 \\
\hline \multicolumn{10}{|l|}{ Idade da mãe } \\
\hline 10 a 17 anos & - & - & - & - & - & - & 2,01 & $1,28-3,15$ & 0,002 \\
\hline 18 a 29 anos & - & - & - & - & - & - & & & \\
\hline \multicolumn{10}{|l|}{ Escolaridade } \\
\hline 1 a 7 anos & 1,23 & $1,02-1,48$ & $\overline{0,03}$ & 1,81 & $1,38-2,39$ & 0 & 1,56 & $1,19-2,06$ & 0,002 \\
\hline
\end{tabular}

Fonte: Pantoja IN, et al., 2021.

\section{Associação entre o número de consultas pré-natal e os desfechos maternos e neonatais}

Após verificarmos que os fatores sociodemográficos maternos influenciam no número de consultas realizadas, de acordo com o município. Passamos então, para análise da influência do número de consultas nos desfechos maternos e neonatais. Os resultados demonstraram associação $(p<0,05)$ do tipo de parto em Bragança e Capanema; o tempo de gestação em todos os municípios; o peso em Bragança e Viseu; e o apgar em Capanema. 
Tabela 3 - Associação entre o número de consultas pré-natal e os desfechos maternos e neonatais nos municípios de Bragança, Capanema e Viseu. 2017.

\begin{tabular}{|c|c|c|c|c|c|c|c|c|c|c|c|c|}
\hline \multirow{4}{*}{$\begin{array}{c}\text { Características } \\
\text { sociodemográficas } \\
\text { maternas }\end{array}$} & \multicolumn{12}{|c|}{ Número de consultas por município } \\
\hline & \multicolumn{4}{|c|}{ Bragança (2099) } & \multicolumn{4}{|c|}{ Viseu (1140) } & \multicolumn{4}{|c|}{ Capanema (1040) } \\
\hline & 0 a 3 & 4 a 7 & $x^{2}$ & $\boldsymbol{P}$ & 0 a 3 & 4 a 7 & $x^{2}$ & $p$ & 0 a 3 & 4 a 7 & $x^{2}$ & $p$ \\
\hline & 744 & 1355 & & & 293 & 847 & & & 400 & 640 & & \\
\hline \multicolumn{13}{|l|}{ Tipo de parto } \\
\hline Vaginal & 451 & 738 & & & 224 & 611 & & & 231 & 258 & & \\
\hline Cesáreo & 293 & 615 & 6.96 & 0.0083 & 62 & 230 & 3.28 & 0.0699 & 166 & 376 & 29.26 & $<0.0001$ \\
\hline $\mathrm{SI}^{*}$ & & 2 & & & 7 & 6 & & & 3 & 6 & & \\
\hline \multicolumn{13}{|l|}{ Tempo de gestação } \\
\hline A termo & 296 & 1002 & & & 116 & 601 & & & 75 & 483 & & \\
\hline $\begin{array}{c}\text { Pré-termo / Pré- } \\
\text { maturo }\end{array}$ & 88 & 152 & 20.04 & $<0.0001$ & 50 & 114 & 16.94 & $<0.0001$ & 25 & 89 & 4.73 & 0.0295 \\
\hline$S I^{*}$ & 360 & 201 & & & 127 & 132 & & & 300 & 68 & & \\
\hline \multicolumn{13}{|l|}{ Peso } \\
\hline$\geq 2500$ gramas & 672 & 1280 & & & 266 & 804 & & & 353 & 584 & & \\
\hline$<2500$ gramas & 71 & 75 & 11.36 & 0.0007 & 26 & 40 & 6.13 & 0.0132 & 47 & 56 & 2.15 & 0.1418 \\
\hline$S I^{*}$ & 1 & & & & 1 & 3 & & & & & & \\
\hline \multicolumn{13}{|l|}{ Apgar $1^{\circ}$ minuto } \\
\hline Normal & 645 & 1245 & & & 198 & 634 & & & 352 & 534 & & \\
\hline Dificuldade & 58 & 81 & 2.97 & 0.0845 & 22 & 47 & 1.84 & 0.1749 & 43 & 100 & 4.45 & 0.0348 \\
\hline $\mathrm{SI}^{*}$ & 41 & 29 & & & 73 & 166 & & & 5 & 6 & & \\
\hline
\end{tabular}

Fonte: Pantoja IN, et al., 2021.

Gestantes que realizaram acima de 4 consultas tem mais chances de ter parto cesáreo que as mulheres que realizaram abaixo de três consultas. Mulheres que realizaram entre 0 e 3 consultas tem mais chances de ter parto pré-termo/prematuro. $O$ baixo peso ao nascer está associado ao menor número de consultas em Viseu e Bragança; enquanto RN que apresentaram qualquer grau de dificuldade está relacionado ao maior número de consultas em Capanema.

Tabela 4 - Regressão logística binária entre o número de consultas pré-natal e os desfechos maternos e neonatais nos municípios de Bragança, Capanema e Viseu. 2017.

\begin{tabular}{|c|c|c|c|c|c|c|c|c|c|}
\hline \multirow{2}{*}{ Variáveis } & \multicolumn{3}{|c|}{ 6+Bragança } & \multicolumn{3}{|c|}{ Viseu } & \multicolumn{3}{|c|}{ Capanema } \\
\hline & $\mathrm{OR}^{*}$ & IC 95\%* & $P$ & OR & IC 95\% & $p$ & OR & IC 95\% & $P$ \\
\hline \multicolumn{10}{|l|}{ Tipo de parto } \\
\hline Cesáreo & 0,78 & $0,65-0,94$ & 0.007 & - & - & - & 0,49 & $0,38-0,64$ & 0 \\
\hline \multicolumn{10}{|c|}{ Tempo de gestação } \\
\hline Prematuridade & 1,96 & $1,46-2,63$ & 0 & 2,27 & $1,54-3,35$ & 0 & 1,81 & $1,09-3,0$ & 0,022 \\
\hline \multicolumn{10}{|l|}{ Peso ao nascer } \\
\hline$<2500$ gramas & 1,8 & $1,29-2,53$ & 0,001 & 1,96 & $1,18-3,28$ & 0,01 & - & - & - \\
\hline \multicolumn{10}{|l|}{ Apgar 1 minute } \\
\hline Dificuldade & - & - & & - & - & - & 0,65 & $0,45-0,96$ & 0,028 \\
\hline
\end{tabular}

Fonte: Pantoja IN, et al., 2021.

\section{DISCUSSÃO}

No presente estudo, em relação as características sociodemográficas maternas observou-se um maior número de mulheres solteiras, discordando de outras pesquisas realizadas no nordeste e sudeste brasileiro que manifestaram, no qual a minoria das mulheres não vive com companheiro (MENDES R, et al., 2019; OLIVEIRA J, et al., 2019). Com referência a escolaridade, foi notabilizado a predominância de 11 anos de estudo, o que são concordantes com os estudos realizados em outras regiões brasileiras os quais apontam o prevalecimento de mulheres com escolaridades acima de 10 anos (MENDES C, et al., 2019). 
Quanto ao número de consultas de pré-natal a maioria tiveram entre 4 a 6 consultas, o que vai de acordo com os resultados obtidos em um estudo realizado no Nordeste Brasileiro (MENDES R, et al., 2019). Porém não são concordantes aos estudos semelhantes realizados em outras regiões brasileiras, como refere Mendes C (2019) o qual prevaleceu mais de 6 consultas de pré-natal. A realização de um bom pré-natal deve acontecer, no mínimo durante 06 consultas, conforme o Ministério da Saúde, devido ao processo fisiológico e psicológico em que essas mulheres passarão a cada trimestre, garantindo assim qualidade de vida, como minimização de partos prematuras, diminuição de transmissão de Infecções Sexualmente Transmissíveis, entre outros (MINISTÉRIO DA SAÚDE, 2016).

Diante dos resultados, podemos visualizer que a Atenção Primária a Saúde (APS), se mostra na saúde maternal como um pilar para uma qualidade na atenção a saúde. A alta cobertura da APS nos municipios permite, que um bom pré-natal seja feito, possibilitando o uso dos serviços disponiveis pelo SUS, como os exames de rotina, as sorologias para detecção de IST's, acompanhamento do crescimento e desenvolvimento do feto, entre outros, refletindo em bons indicadores na redução da mortalidade maternal e perinatal (LEAL M, et al., 2018).

Em relação ao tempo de gestação a maior parte teve gestação entre 37 a 41 semanas, semelhantes aos achados de outros estudos brasileiros que vão de acordo com o tempo de gestação da análise (MENDES R, et al., 2019; OLIVEIRA J, et al., 2019; REGINATTO P, et al., 2017).

Quando analisados o tipo de parto, a preeminência do estudo foi de partos vaginais, o que testifica com a literatura encontrada, onde a maioria foram de parto normais. Entretanto, em outros dados obtidos por outros autores no Brasil, foi observado uma prevalência de parto cesáreo, o que contrapõe ao estudo realizado (OLIVEIRA J, et al., 2019; MENDES C, et al., 2019; BRASIL, et al., 2019).

Em relação ao Apgar, a maioria apresentou uma classificação como ótimo Apgar no primeiro minuto, similar a análise realizada no sul do Brasil. Ao inverso destes estudos, foram achados por autores um estudo no nordeste onde o maior número da classificação foi $<7$. Dentre o estudo foram detectados que a maioria dos recém-nascidos apresentaram o peso acima de 2.500 , o que foi semelhante à relatada em um estudo prospectivo identificado há prevalência de recém-nascidos acima de 2500 (REGINATTO P, et al., 2017; BRASIL, et al., 2019).

Com relação ao número de consultas de pré-natal, os achados do presente estudo são discordantes aos estudos semelhantes realizados em outras regiões brasileiras, os quais apontaram associação entre as adolescentes com o menor número de consultas pré-natal (GRAVENA F, et al., 2013).

Houve associação significantes entre o número de consultas e a faixa etária materna entre 10 a 17 anos, discordando com dados de outras pesquisas realizadas em outras regiões do país, em que não houve associação significantes. Em relação ao número de consultas com o tipo de parto houve uma associação em que 04 consultas foram associadas com maiores chances de ter cesárea, diferente dos achados em outra pesquisa, em que o maior número de consultas foi proporcional a partos vaginais (SANTOS C, et al., 2014).

Quanto a relação entre a associação entre características sociodemográficas maternas e o número de consultas pré-natal, foi classificado um menor número de consultas no pré-natal com o baixo peso e prematuridade observado no estudo, discorda com dados de outras pesquisas realizadas em outra região do país, o qual apontou que o menor número de consultas não conferiu uma chance aumentada de ocorrência de prematuridade e baixo peso ao nascer (SANTOS C, et al., 2014). Em um outro estudo houve uma associação entre o maior número de consultas e maior o número de partos vaginais, além de peso adequado, o que corrobora com os achados na pesquisa (BORGES M, et al., 2019)

É evidenciado nas pesquisas, que os resultados maternos e neonatais refletem as condições em que a mulher está inserida, além da qualidade dos serviços disponíveis que são ofertados, visto que para se ter realizar um bom pré-natal, deve-se seguir as diretrizes e recomendações do Ministério da saúde, desde o preenchimento da caderneta, contendo as principais informações do que foi realizado na assistência até as mínimas informações dias antes da data provável do parto. 
Isso então, reflete na continuidade da assistência, nas carcateristicas maternos e neonatais, pois diante desses resultados relacionados aos achados na literatura científica, permite-se refletir sobre as mudanças de práticas nos serviços públicos de saúde, principalmente pelo fato de encontramos frequentemente um perfil de mulheres com baixo nível sócio-economico, que apresentam na maioria das vezes, gravidez não planejada.

\section{CONCLUSÃO}

Os resultados demonstram que a assistência pré-natal é significativo no momento do parto e pós-parto, pois apresenta um propósito de detectar situações de risco, reduzindo a ocorrência de morbidade e mortalidade materna e neonatal, levantando a discussão de capacitação de profissionais da APS (Atenção Primária a Saúde), que representam o primeiro contato com a mulher que está iniciando seu pré-natal. Esses resultados denotam que um acompanhamento pré-natal de qualidade pode ajudar na identificação precoce de intercorrências gestacionais contribuindo na redução de riscos tanto para a gestante quanto para o concepto, tornando-o imprescindível para a promoção da saúde materna e neonatal, fazendo-se um indispensável investimento em estratégias que viabilizem novos estudos acerca do tema em questão.

\section{REFERÊNCIAS}

1. ARAUJO E, et al. Importância do pré-natal na prevenção da sífilis congênita. Rev Para Med. 2017; 20:47-51.

2. BORGES E, et al. Condição Materna de Adolescentes e Impactos no Peso do Neonato. Revista Brasileira de Educação e Saúde, 2019, 9; 43-49.

3. DARMONT M, et al. Adesão ao pré-natal de mulheres HIV+ que não fizeram profilaxia da transmissão vertical: um estudo sócio-comportamental e de acesso ao sistema de saúde. Cad Saude Publica, 2015, 26:1788-96.

4. FESCINAR R, et al. Salud sexual y reproductiva: guías para el continuo de atención de la mujer y el recién nascido focalizadas en la APS. 3.ed. Uruguay: Montevideo, 2011. Disponível em: https:/www.paho.org/clap/index.php?option=com_docman\&view=download\&alias=202-salud-sexual-y reproductivaguias-para-el-continuo-de-atencion-de-la-mujer-y-el-recien-nacido-focalizadas-en-aps-3ed\&category_slug=publicaciones\&ltemid=219\&lang=en. Acessado em: junho de 2019.

5. FERRAZ L, BORDIGNON M. Mortalidade materna no brasil: uma realidade que precisa melhorar. Rev Bai de Saúde Pub, 2015; 36: e5275.

6. GRAVENA F, et al. Idade materna e fatores associados a resultados perinatais. Acta Paul Enferm. 2013,26 ; 130-5.

7. LEAL M, et al. Saúde reprodutiva, materna, neonatal e infantil nos 30 anos do Sistema Único de Saúde (SUS). Ciência \& Saúde Coletiva, 2018, 23: 1915-1928.

8. MARTINS A, SILVA L. Perfi Epidemiológico de mortalidade maternal. Rev Bras Enfermagem, 2018;71:677-83.

9. MENDES R, et al. Características maternas e da assistência pré-natal associadas à peregrinação no anteparto. Rev Saude Publica, 2019, 10; 53-70.

10. MENDES C, et al. Fatores relacionados com uma menor duração total do aleitamento materno. Ciência \& Saúde Coletiva, 2019, 24; 1821-1829.

11. MINISTÉRIO DA SAÚDE. Protocolos da Atenção Básica: saúde das mulheres. Instituto Sírio-Libanês de Ensino e Pesquisa. $\quad$ Brasil, $2015 . \quad$ Disponível em: http://bvsms.saude.gov.br/bvs/publicacoes/protocolos_atencao_basica_saude_mulheres.pdf. Acessado em: $10 \mathrm{de}$ setembro de 2019.

12. MINISTÉRIO DA SAÚDE. Atenção ao Pré-Natal de Baixo Risco. Brasília - DF, Brasil, 2016. Disponível em: https://bvsms.saude.gov.br/bvs/publicacoes/cadernos_atencao_basica_32_prenatal.pdf. Acessado em: 15 de Fevereiro de 2019.

13. NASCIMENTO I, et al. Assistência Pré-Natal E Resultado Perinatal. Rev Bras Promoç Saúde, 2017, 2; $187-194$.

14. OLIVEIRA J, et al. Resultados perinatais e do primeiro ano de vida segundo cor da pele materna: estudo de coorte. Rev Esc Enferm USP, 2019, 53; 74-80.

15. OLIVEIRA E, et al. A Importância Do Acompanhamento Pré-Natal Realizado Por Enfermeiros. Revista Científica FacMais, 2016, 8; 8-14

16. REGINATTO P, et al. Prevalence and characterization of neonatal skin disorders in the first $72 \mathrm{~h}$ of life. J Pediatr (Rio J), 2017, 93;238-245.

17. SANTOS C, et al. Gravidez na adolescência: análise de fatores de risco para baixo peso, prematuridade e cesariana. Ciência \& Saúde Coletiva, 2014, 19; 719-726.

18. SILVA A, et al. Puerpério remoto: seguimento do primeiro ano pós-parto pela atenção primária de saúde. Revista Eletrônica Acervo Saúde, 2021; 1: 1-10.

19. SOUZA K, et al. Rede cegonha e desafios metodológicos de implementação de redes no SUS. Cien Saude Colet, $2021 ; 26: 176-83$.

20. VIELLAS E, et al. Assistência Pré-Natal no Brasil. Caderno Saúde Pública, 2014,30: 85-100. 